Itinéraires Itinéraires

Littérature, textes, cultures

\title{
Bengt Jangfeldt, La Vie en jeu : une biographie de Vladimir Maïakovski
}

Traduction de Rémi Cassaigne, Paris, Albin Michel, 2010.

\section{Aurore Peyroles}

\section{(2) OpenEdition}

\section{Journals}

Édition électronique

URL : http://journals.openedition.org/itineraires/1443

DOI : $10.4000 /$ itineraires. 1443

ISSN : 2427-920X

Éditeur

Pléiade

Édition imprimée

Date de publication : 1 décembre 2011

Pagination : 173-179

ISBN : 978-2-296-55744-4

ISSN : 2100-1340

\section{Référence électronique}

Aurore Peyroles, « Bengt Jangfeldt, La Vie en jeu : une biographie de Vladimir Maïakovski», Itinéraires [En ligne], 2011-4 | 2011, mis en ligne le 01 décembre 2011, consulté le 22 septembre 2020. URL : http:// journals.openedition.org/itineraires/1443; DOI : https://doi.org/10.4000/itineraires.1443

Ce document a été généré automatiquement le 22 septembre 2020.

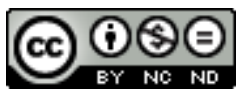

Itinéraires est mis à disposition selon les termes de la licence Creative Commons Attribution - Pas d'Utilisation Commerciale - Pas de Modification 4.0 International. 


\section{Bengt Jangfeldt, La Vie en jeu : une biographie de Vladimir Maïakovski}

Traduction de Rémi Cassaigne, Paris, Albin Michel, 2010.

\section{Aurore Peyroles}

\section{RÉFÉRENCE}

Bengt Jangfeldt, La Vie en jeu : une biographie de Vladimir Maïakovski, Traduction de Rémi Cassaigne, Paris, Albin Michel, 2010.

$$
\begin{array}{r}
\text { Mourir } \\
\text { dans cette vie } \\
\text { n'est pas difficile. } \\
\text { Il est bien plus difficile } \\
\text { de construire sa vie. }
\end{array}
$$

« Difficile de construire sa vie », difficile aussi de construire le récit d'une vie aussi

mouvementée que celle de Vladimir

Vladimirovitch Maïakovski (1893-1930), si l'on en juge par le peu d'ouvrages biographiques qui lui ont été consacrés. Comment comprendre pourtant les textes du grand poète soviétique sans les replacer dans leur contexte, personnel et historique, avec lequel ils entretiennent des liens si étroits? Fort de la conviction qu'une telle recontextualisation était nécessaire et que la vie de Maïakovski constituait en soi un matériau passionnant, fort aussi d'une connaissance personnelle des derniers protagonistes de cette histoire, Bengt Jangfeldt a affronté la complexité de la tâche. Classiquement organisée de façon chronologique, sa colossale enquête entend saisir tous les aspects de l'homme, de sa phobie des microbes à son goût pour les vêtements de luxe, de Moscou à New York, de ses scénarios à ses slogans publicitaires, de son amour pour Lili Brik à ses louanges de Lénine. Contexte historico-politique, ô combien influent dans la trajectoire de Maïakovski, histoires amoureuses (passion unique pour Lili et aventures avec beaucoup d'autres), relations amicales et intellectuelles, parcours 
idéologique et poétique, rien ne manque dans ce livre où abondent les faits. Mais à la recherche de quel Maïakovski s'est lancé son biographe?

Commençons par la fin: le 14 avril 1930, Maïakovski se suicide dans son studio moscovite. Cette mort volontaire prend immédiatement les proportions d'une affaire d'État : quel embarras pour le pouvoir soviétique de voir son poète le plus connu, celui qui a accompagné de ses textes les moments marquants de sa courte histoire, mettre fin à ses jours, en plein premier plan quinquennal... Comment cet acte sera-t-il perçu, à l'intérieur et à l'extérieur de l'Union? Comment forger un récit convaincant qui expliquera ce qui doit aussi rester inexplicable - renoncer à la vie dans le pays qui promettait une vie nouvelle? Dès le lendemain de sa mort, les autorités livrent une version officielle de l'événement : le grand poète s'est tué "pour une bonne femme ", les raisons en sont uniquement privées. La biographie de Maïakovski est désormais entre les mains de l'État soviétique. Mise en abyme du rôle capital de l'entreprise biographique: il s'agit toujours de façonner une interprétation de trajectoires individuelles, de livrer une interprétation d'une trajectoire individuelle. Le récit officiel ne convaincra pas les amis du poète, prompts au contraire à voir dans ce suicide un défi lancé au pouvoir stalinien et une condamnation de sa politique littéraire. Mais il aura des conséquences durables: Maïakovski est érigé en mythe. Biographie et œuvre poétique sont revues à l'aune de cette nouvelle canonisation, les textes politiques prennent le pas sur les poèmes lyriques. La terreur du poète de se voir transformer en monument est réalisée, au-delà probablement de ses craintes. Littéralement d'abord : la place qui portait son nom à Moscou est dominée par une colossale statue le représentant; littérairement ensuite: une fois mort, Maïakovski connaît "une diffusion obligatoire, comme la pomme de terre sous Catherine II ", pour reprendre l'expression d'un Pasternak consterné1. Il est devenu le poète d'Octobre et du régime bolchevique - à tel point qu'il sera supprimé de l'enseignement scolaire après la chute de ce dernier : poète obligatoire, il en devenait indésirable.

3 Le grand mérite de l'ouvrage de Bengt Jangfeldt est de déboulonner ce Maïakovski monumentalisé et figé dans la légende soviétique : elle se conçoit comme l'écriture de la «vraie » vie de Maïakovski - et en particulier de tous ses aspects occultés par la version officielle. C'est la raison pour laquelle l'auteur donne tant d'importance à Lili Brik, objet à part égale de cette biographie : il s'agit de rendre au poète soviétique sa vie amoureuse tumultueuse, au cœur de nombre de ses poèmes dont le lyrisme est toujours autobiographique. Au lieu de l'épopée, le biographe préfère écrire le roman - d'amour, d'aventures, de formation - que constitue à ses yeux la vie de Maïakovski. Ce choix influence ses partis pris narratifs: une narration au présent qui dramatise les trajectoires, des images fortes - la toute jeune Lili Brik parcourant les vastes étendues du Turkestan, le bruit des talons ferrés de Maïakovski résonnant sur Broadway. Pas de notes de bas de page ni de statistiques, mais de nombreux extraits de correspondances intimes et de nombreuses photographies qui donnent à l'ouvrage un air d'album de famille : le lecteur est introduit dans le microcosme du deux-pièces des Brik où amis, parents et amants se rencontrent et où s'écrit une part de l'histoire soviétique.

4 Retrouver le révolté derrière le révolutionnaire et le poète derrière le propagandiste, telle est l'ambition de Bengt Jangfeldt. Il y parvient, incontestablement. Et même trop bien. S'efforçant de renverser un récit officiel privilégiant la sphère politique, il consacre l'essentiel de sa biographie à la sphère sentimentale, au détriment, non du contexte historique, mais du caractère politiquement engagé de l'existence et de la 
poésie de Maïakovski. L'ouvrage s'ouvre sur la promesse d'évoquer les «orages politiques, littéraires et privés » qui ont rythmé la vie du poète soviétique; mais cette triade cède la place à l'évocation, avec force détails, du trio que formaient le poète, son amante Lili et le mari de cette dernière, Ossip Brik. La vie sentimentale du poète finit par éclipser quelque peu les autres centres de gravité de son existence.

5 Ainsi, le lecteur ne pourra être que frappé du contraste entre la place accordée aux amours du poète et le peu de cas qui est fait de ses rapports avec le pouvoir soviétique et de ses convictions politiques. Pourtant, que de revirements, de part et d'autre! Au lendemain de la prise de pouvoir par les bolcheviques, Maïakovski lance, en tant que porte-parole de l'Union des travailleurs culturels : « Ma devise, et c'est la nôtre à tous : Vive la vie politique russe et vive un art indépendant de l'État », avant de préciser : " Je ne dis pas non à la politique, mais dans l'art il n'y a pas de place pour la politique. » Conception aux antipodes de celle du nouveau pouvoir, qui entend au contraire intervenir dans la vie culturelle du pays et se montre très vite soucieux de définir les formes que doit revêtir l'art soviétique. La norme réaliste, établie bien avant sa formalisation sous l'étiquette du réalisme socialiste, prévaut, aux dépens de toute expérimentation formelle ${ }^{2}$ - quand bien même cette dernière se proposait d'agir "utilement». "Aucun écrivain n'est aussi indissociable de la Révolution russe que Maïakovski », prévenait l'introduction de la biographie. La suite de l'ouvrage montre pourtant qu'il ne fut aucunement le poète tout désigné d'octobre 1917, ni de sa part ni de celle du pouvoir. En témoigne la réaction de Lénine à 150000 000, le grand poème épique que Maïakovski écrit à la gloire de la Révolution :

N'est-ce pas une honte de voter la publication à 5000 exemplaires du 150000000 de Maïakovski? Foutaises, stupidités, idiotie crasse et prétentieuse. À mon avis, il ne faut imprimer les choses de ce genre qu'une fois sur dix, et pas à plus de 1500 exemplaires, c'est bien assez pour les bibliothèques et les tordus ${ }^{3}$.

6 La vie de Maïakovski et de ses comparses futuristes ne suit pas une courbe progressant de l'euphorie révolutionnaire à la dépression stalinienne: les difficultés dans leurs rapports au pouvoir politique, marqués par une méfiance réciproque, ont été constantes; à aucun moment le futurisme n'a obtenu le statut d'art officiel ni été adoubé par l'idéologie étatique.

7 Or, si Bengt Jangfeldt insiste à juste titre sur la défiance des instances officielles à l'égard des futuristes, il accorde bien peu de place à la volte-face de Maïakovski et de beaucoup d'autres qui choisissent de se rallier entièrement au régime bolchevique. Car le poète se "soumet " et se "convertit» à "l'art utile » - ce sont les termes du biographe. Cet effort - vain - de reconnaissance auprès du pouvoir n'est d'ailleurs pas sans faire penser aux relations qui unissent le poète et sa muse, Lili Brik: relations mouvementées pour le moins, où s'opposent une farouche persévérance à se faire aimer et un rejet de la part de l'objet désirét. Mais comment l'expliquer? Serait-ce que Maïakovski et ses amis n'étaient pas si étrangers à la politique que la biographie semble l'indiquer? Ou que le sujet politique s'imposait de lui-même? Ces questions se posent, car le retournement de Maïakovski n'est pas pure formalité. Son soutien au pouvoir est infaillible, jusqu'à la fin ${ }^{5}$. Même dans le contexte des répressions de plus en plus ouvertes de la fin des années 1920 (épuration des opposants, collectivisation forcée, procès spectacles), il persiste à donner raison au gouvernement - contrairement à Pasternak, par exemple, qui quitte ce qui reste du groupe futuriste, rebuté par «sa prédilection pour la révolte avec un mandat officiel de révolte en poche ». 
Plus fondamentalement encore, Maïakovski modifie sa pratique poétique: la contestation formelle de ses débuts futuristes s'allie au registre politique. Le poète ne chante plus seulement la cruauté de la femme aimée ni ses rêves d'absolu, mais les louanges de Lénine ${ }^{6}$ et de la Tchéka. Comment ne pas penser à l'Aragon de Front rouge et $\mathrm{du}$ Prélude aux temps des cerises que rythme le refrain "Vive le Guépéou "?

? Les deux poètes mettent au service de leur nouvelle « famille » ou " patrie », c'est selon, l'extraordinaire efficacité de leur langue poétique ainsi que leur insolente facilité à manier - à manipuler - le langage. Tous deux seront d'ailleurs amenés à rompre avec leurs amitiés premières, fondées sur une conception de la littérature antagoniste à celle

qu'ils adoptent, plus ou moins volontairement - tout en ayant beaucoup de mal à convaincre leurs nouvelles relations du sérieux de leur engagement. Ils s'efforceront d'approcher leur public rêvé, nécessairement prolétaire, par l'organisation de lectures publiques au cours desquelles ils sont confrontés au reproche inlassablement reformulé de leur illisibilité - il semble que Maïakovski ait bien davantage apprécié et pratiqué l'exercice. Celui qui proclamait que « dans l'art il n'y a pas de place pour la politique » et celui qui vomissait « Moscou la gâteuse » sont devenus les plus grands poètes politiques du $\mathrm{xx}^{\mathrm{e}}$ siècle.

9 La biographie de Bengt Jangfeldt retrace cette évolution de Maïakovski et de son art, d'un apolitisme revendiqué à un engagement aux côtés du pouvoir qui dépassait de loin ce qu'on attendait de lui. Mais ce spectaculaire revirement reste inexpliqué. Peur? Soif de reconnaissance? Conversion idéologique ? Jangfeldt ne tranche pas ni ne s'y attarde, se contentant de remarquer: "Maïakovski n'était ni opportuniste ni cynique, mais politiquement naïf et, dans son désir de construire une société nouvelle et meilleure, complètement aveugle aux événements qui dans la réalité allaient à l'encontre d'un tel développement.» La "naïveté politique» du poète expliquerait-elle donc son engagement, public et poétique, aux côtés du pouvoir bolchevique? Bengt Jangfeldt insiste pourtant plus loin sur les remords de Maïakovski, soudainement conscient d'être devenu un fonctionnaire $^{8}$ ». C'est le silence le plus retentissant de cette biographie : quelles étaient les convictions politiques de Maïakovski? Quel jugement portait-il sur le régime qu'il soutenait à voix si haute? Le tout jeune Vladimir avait adhéré au Parti bolchevique dès l'âge de quinze ans - activité militante qui lui valut trois arrestations. Que restait-il de cet engagement si précoce chez le poète futuriste? Que le biographe ne puisse répondre à ces interrogations, cela ne saurait lui être reproché. On regrettera pourtant que l'engagement politique ne soit envisagé de sa part que comme une compromission morale et littéraire.

Le dénigrement de l'activité militante de Maïakovski interdit en effet au biographe de poser la question essentielle de l'influence politique sur sa pratique poétique : comment ce dernier écrit-il la politique? Comment ce nouvel objet exerce-t-il un effet en retour sur sa poésie? Cela ne fait l'objet d'aucune analyse, et c'est fort dommage ${ }^{9}$. Ce silence reflète certainement la certitude du biographe qu'il ne saurait exister de poésie politique : il n'hésite pas en effet, à propos des poèmes d'actualité ou de circonstance de Maïakovski, à parler de "négation autodestructrice de la poésie ». « Même s'il refuse de le reconnaître ouvertement, Maïakovski est tourmenté par l'idée que la production constante de vers de circonstance puisse l'empêcher d'écrire de la vraie poésie ", écrit encore Bengt Jangfeldt. Opposer une "fausse » et une "vraie» poésie paraît bien hasardeux, et l'absence avouée de sources permet de renvoyer ces affirmations à la 
conviction du seul biographe. La constante antinomie qu'opère le biographe entre poésie lyrique amoureuse, splendide et révolutionnaire, et poésie politique, marquée par ce qu'il considère être l'inévitable médiocrité du genre, paraît bien trop franche au regard de l'intrication revendiquée par le poète lui-même dans ces vers : « Des cieux de la poésie, je descends vers le communisme parce que, sans le communisme, il n'est pas pour moi d'amour. » Claude Frioux a au contraire souligné l'unité de l'inspiration et de l'œuvre maïakovskiennes :

La quête d'une vie «étonnante » où tout soit «agrandi, redessiné » et intensifié, dont l'amour lui a révélé la nostalgie sans la satisfaire, est la motivation profonde des penchants cosmiques et fantastiques de son imagination visionnaire [...] de même que celle de son adhésion passionnée au communisme dont il attendait avant tout une métamorphose féerique de l'existence ${ }^{10}$.

11 Dans son dernier discours, Maïakovski se moquait «[d]es esthètes [qui l']engueulent : "Vous écriviez de si beaux vers, leNuage en pantalon, et brusquement, vous vous mettez à faire de pareilles choses !"11 "; son biographe en est parfois bien proche...

12 Au mythe du poète prolétarien, forgé par le pouvoir soviétique de façon posthume, succède, sous la plume de Bengt Jangfeldt, celui d'un poète à la veine lyrique contrarié : une légende romantique naît des ruines de la légende bolchevique. Comme si la complexité de la vie et de la poésie de Maïakovski devait échapper à l'entreprise biographique. La richesse de la documentation et des témoignages de première main, la vivacité de la narration de cette biographie sont remarquables. Mais à force de dissocier art et politique, deux domaines que Maïakovski s'est pourtant efforcé de mêler dans une pratique poétique renouvelée, Jangfeldt dessine surtout la figure d'un amoureux toujours déçu et d'un joueur jamais assuré de gagner, retrouvant l'homme plutôt que le poète. Or Jakobson n'avait-il pas déjà livré la meilleure caractérisation de son ami de jeunesse avec ce commentaire laconique : « [Maïakovski] était une personne particulièrement mal équipée pour cette vie »?

\section{NOTES}

1. Des années plus tard, en 1968, Elsa Triolet fait le même constat: «Ayant perdu la bataille contre sa poésie de son vivant, les détracteurs de Maïakovski ont inventé de l'annexer. Ce fondateur du futurisme russe, [...] ce bagarreur forcené pour l'art d'avant-garde devient, sous la plume de ses biographes et nombreux mémorialistes, un doux agneau, un sage élève de patronage, une victime de son entourage fait de bandits d'avant-garde tels que Brik, Pasternak, Eisenstein, Meyerhold, Babel, Malevitch, Tatline... Bref toute la fine fleur de l'art soviétique que Maïakovski a su attirer dans sa revue Lef » (Elsa Triolet, « Préface », Grand Jamais, citée par Pierre Daix, Avec Elsa Triolet (1945-1971), Paris, Gallimard, 2010, p. 196).

2. Cette interprétation de la politique culturelle bolchevique qui remet en cause la distinction habituellement opérée entre les premières années de liberté artistique et la période stalinienne imposant des normes et des obligations (comme le fait Régine Robin dans son ouvrage de référence, Le Réalisme socialiste: une esthétique impossible, Paris, Payot, 1986) correspond aux recherches les plus récentes. Voir à ce propos la première partie de la thèse de Reynald Lahanque 
Le Réalisme socialiste en France : 1934-1954 (sous la direction de Guy Borreli, Université Nancy 2, 2002).

3. Lénine, cité par Bengt Jangfeldt, La Vie en jeu, op. cit., p. 167. La citation est extraite d'une lettre adressée à Lounatcharski le 6 mai 1921.

4. Lili a toujours été plus séduite par le poète que par l'homme : «J'ai tout de suite compris que Volodia était un poète génial, mais il ne me plaisait pas. [...] Je n'aimais même pas son nom Maïakovski - tellement clinquant qu'on aurait dit un pseudonyme, et un pseudonyme vulgaire en plus » (maiak signifiant « phare » en russe). Elsa, qui présenta Maïakovski aux Brik, se rappelle : «Les Brik furent transportés par les poèmes et en tombèrent amoureux irrévocablement, [et Maïakovski] tomba irrévocablement amoureux de Lili. » Le seul statut que Lili entendait occuper sans partage était celui de la muse du poète, non celui de l'amante. Elle se livrera à des manigances peu recommandables dès que ce statut était menacé par d'autres femmes aimées de Maïakovski.

5. Il n'est qu'un domaine où Maïakovski ne s'est pas efforcé de se conformer à ce qu'exigeait son rang de poète soviétique : sa vie amoureuse et mondaine. À Paris, il côtoie les cercles de Russes émigrés, jusqu'à demander en mariage une compatriote qui a fui la Russie bolchevique - leur relation fera l'objet d'une étroite surveillance par les services secrets soviétiques... et par Lili. Étonnante URSS des années 1920 où persistent les privilèges sociaux, le goût du luxe et des voyages.

6. Dans Vladimir Ilitch Lénine, par exemple, 3000 vers épiques écrits en hommage à la mort du premier dirigeant de l'URSS, dédiés à la classe ouvrière : «Toute / ma force / sonnante de poète / je t'en fais don, / classe qui passe à l'offensive / Prolétariat ».

7. Aragon est très peu évoqué dans cette biographie de Maïakovski. Malgré la mention de plusieurs rencontres à Paris, rien n'est dit sur le jugement porté par l'un sur l'autre. C'est une des frustrations qu'on éprouve à la lecture de cet ouvrage: le manque d'archives ou les silences «politiquement corrects» des protagonistes empêchent souvent d'approcher des aspects essentiels. Le lecteur français remarquera également que Bengt Jangfeldt est moins familier du contexte français que soviétique. Ainsi fait-il remonter le mariage d'Aragon et d'Elsa Triolet à 1930, alors qu'ils ne se marieront qu'en 1939. Surtout, il affirme qu'Elsa fut... un «membre éminent du PCF », alors qu'elle n'y adhéra jamais.

8. Annenkov, un poète russe exilé à Nice, raconte au biographe sa dernière entrevue avec Maïakovski : quand ce dernier lui demande s'il pense rentrer à Moscou, Annenkov lui répond que, puisqu'il entend rester un artiste, il n'en a pas l'intention. « Maïakovski m'a donné une tape sur l'épaule, il s'est soudain assombri et a dit d'une voix rauque: "Moi j'y retourne... puisque je ne suis plus poète ?" Puis une scène dramatique : Maïakovski a éclaté en sanglots et a chuchoté, à peine audible : “À présent, je suis... fonctionnaire...” ".

9. On regrettera plus généralement l'absence de toute perspective littéraire : malgré la citation de larges extraits de textes maïakovskiens, pas un mot sur le démantèlement du vers, pas un mot non plus sur les innovations formelles ou langagières. Les vers du poète ne sont convoqués que dans une perspective biographique.

10. Claude Frioux, dans l'article de l'Encyclopedia Universalis consacré à Maïakovski.

11. Discours du 25 mars 1930, cité par Elsa Triolet dans Souvenirs sur Maïakovski, Paris, Les Éditeurs français réunis, 1957, p. 46. 


\section{AUTEURS}

\section{AURORE PEYROLES}

Université Paris 13 - CENEL 